\title{
ON THEOREMS OF MORGAN AND COWLING-PRICE FOR SELECTED NILPOTENT LIE GROUPS
}

\author{
KAIS SMAOUI
}

Abstract. Let $G$ be a connected, simply connected nilpotent Lie group. For $p, q \in[1,+\infty]$, the $L^{p}-L^{q}$ analogue of Morgan's theorem was proved only for two step nilpotent Lie groups. In order to study this problem in larger subclasses, we formulate and prove a version of $L^{p}-$ $L^{q}$ Morgan's theorem on nilpotent Lie groups whose Lie algebra admits an ideal which is a polarization for a dense subset of generic linear forms on the Lie algebra. A proof of an analogue of Cowling-Price Theorem is also provided in the same context.

Mathematics subject classification (2010): Primary 22E25, Secondary 43A25.

Keywords and phrases: Uncertainty principle, Fourier transform, Plancherel formula.

\section{REFERENCES}

[1] F. Abdelmoula, And A. Baklouti, The $L^{p}-L^{q}$ analogue of Morgan's theorem on exponential solvable Lie groups, Math. Notes 88, 4 (2010), 464-478.

[2] S. AYADI AND K. MoKNI, An $L^{p}-L^{q}$-version of Morgan's theorem for the $n$-dimensional euclidean motion group, International Journal of Mathematics and Mathematical Sciences, 2007, 1 (2007).

[3] A. Baklouti, K. Smaoui And J. Ludwig, Estimate of $L^{p}$-Fourier transform norm on nilpotent Lie groups, J. Funct. Anal. 199, 2 (2003), 508-520.

[4] A. Baklouti, N. Ben Salah, And K. Smaoui, Some uncertainty principles on nilpotent Lie groups, Contemporary Mathematics, 363 (2004), 39-52.

[5] A. BAKLOUTI AND N. BEN SALAH, The $L^{p}-L^{q}$ version of Hardy's Theorem on nilpotent Lie groups, Forum Math. 18, 2 (2006), 245-262.

[6] A. Baklouti And S. Thangavelu, Variants of Miyachi's Theorem for nilpotent Lie groups, J. Aust. Math. Soc. 88 (2010), 1-17.

[7] S. BEN FARAH AND K. MOKNI, Uncertainty principle and the $L^{p}-L^{q}$-version of Morgan's theorem on some groups, Russ. J. Math. Phys. 10, 3 (2003), 245-260.

[8] M. Cowling, A. SitARAm AND M. Sundari, Hardy's uncertainty principle on semi simple groups, Pacific J. Math. 192 (2000), 293-296.

[9] M. COWling And J. F. PRICE, Generalisations of Heisenberg's inequaliy, in Harmonic analysis (Cortona, 1982), 443-449, Lecture Notes in Math. 992, Springer, Berlin.

[10] L. CORWIN, AND F. P. GREENLEAF, Representations of nilpotent Lie groups and their applications, Part 1: Basic theory and examples, Cambridge University Press, 1990.

[11] G. H. HARDY, A theorem concerning Fourier transforms, J. London Math. Soc. 8 (1933), 227-231.

[12] E. Kaniuth And A. Kumar, Hardy's Theorem for nilpotent Lie groups, Math. Proc. Camb. Philos. Soc. 131 (2001), 487-494.

[13] G. W. Morgan, A note on Fourier transforms, J. London Math. Soc. 9 (1934), 187-192.

[14] S. PARUi, AND R. P. SARKaR, Beurling's theorem and $L^{p}-L^{q}$ Morgan's theorem for step two nilpotent Lie groups, Publ. RIMS, Kyoto Univ. 44 (2008), 1027-1056.

[15] S. K. RAY, Uncertainty principles on two step nilpotent Lie groups, Proc. Indian Acad. Sci. (Math. Sci.), 111, 3 (2001), 293-318.

[16] J. SEnGUPTA, The uncertainty principle on Riemannian symmetric spaces of the noncompact type, Proceedings of the American Mathematical Society, 130, 4 (2001), 1009-1017. 